\title{
Realizing isomorphisms of category algebras
}

\section{Dorothy Maharam and A.H. Stone}

\author{
Let $\mathcal{C}(X)$ denote the complete boolean algebra of Borel sets \\ modulo first category sets of the space $X$. Given an \\ isomorphism $\tau$ between $C(X)$ and $C(Y)$, where $X$ and $Y$ are \\ complete metric spaces, it is shown that there exists a \\ homeomorphism $T$, between residual subsets $A$ of $X$ and $B$ of \\ $Y$, that induces $\tau$. When $X=Y$ one can make $A=B$. An \\ analogous result is stated when $\tau$ is a complete isomorphism \\ on to a subalgebra.
}

A well-known property of the (Lebesgue) measure algebra, of measurable sets modulo null sets, on (say) a Cantor set of positive measure, is that every automorphism of it can be realized by a measurable point-mapping of the space (see $[4,1]$ ). Some time ago, Kakutani asked us whether, analogously, every automorphism of the "category algebra", of Borel sets modulo first category sets, of the Cantor set can be realized by a homeomorphism of the space. Taken literally, the answer is "no", as is shown by an example in [3, p. 130]. But it is reasonable here to permit the deletion of a first category set - or (what comes to the same thing) to replace "homeomorphism" by "bijection preserving both borelness and first category, both ways". Now, as we show below, the answer is "yes", not merely for the Cantor set but for all (not necessarily separable) complete metric spaces.

Before stating the theorem we observe that, if $A$ is a dense $G_{\delta}$ subset (or more generally a residual subset) of a complete metric space

Received 4 May 1978. 
$X$, then there is a natural isomorphism between the category algebras $\mathcal{C}(X), \mathcal{C}(A)$. (Merely map the class, modulo first category sets, of a Borel set $H$ of $X$, to the class of $H \cap A$ modulo first category subsets of $A$.$) Thus if X, Y$ are complete metric spaces having dense $G_{\delta}$ subsets $A, B$ respectively, and if there is a homeomorphism $T$ of $A$ onto $B$, then $T$ induces in an obvious way an isomorphism $\tau$ of $\mathcal{C}(X)$ onto $C(Y)$. Our main result asserts that, conversely, every such isomorphism arises in this way.

THEOREM. Let $X, Y$ be complete metric spaces, with isomorphic category algebras $\mathcal{C}(X), \mathcal{C}(Y)$, and suppose an isomorphism $\tau$ of $\mathcal{C}(X)$ onto $\mathcal{C}(Y)$ is given. Then there exist dense $G_{\delta}$ subsets $A$ of $X, B$ of $Y$, and a homeomorphism $T$ of $A$ onto $B$, such that $T$ induces $\tau$.

Proof. As is well-known (see [2, Chapter 4]), for every Baire space $Z, C(Z)$ is isomorphic to the complete boolean algebra $R(Z)$ formed by the regular open subsets of $Z$ : each Borel set in $Z$ differs by a first category set from exactly one regular open set, giving the isomorphism. (Note that in $R(Z)$ we have $G \wedge H=G \cap H$ but

$$
\left.\vee\left\{G_{\lambda}: \lambda \in \Lambda\right\}=\operatorname{int}\left(\mathrm{cl} \cup\left\{G_{\lambda}: \lambda \in \Lambda\right\}\right) .\right)
$$

Thus $\tau$ determines an isomorphism $\theta$ of $R(X)$ onto $R(Y)$, and it is convenient to work with $\theta$ rather than $\tau$.

Let $\rho, \sigma$ be complete metrics for $X, Y$ respectively. Take a maximal pairwise disjoint collection $u_{1}$ of nonempty regular open subsets of $X$, all of $\rho$-diameters less than 1 ; say (to avoid suffixes on suffixes) $U_{1}=\left\{U\left(\lambda_{1}\right): \lambda_{1} \in \Lambda_{1}\right\}$. Define $G_{1}=U U_{1}$ : then (from maximality) $\bar{G}_{1}=X$, so $v u_{1}=X$. Put $V\left(\lambda_{1}\right)=\theta\left(U\left(\lambda_{1}\right)\right) \in R(Y)$, and $V_{1}=\left\{V\left(\lambda_{1}\right): \lambda_{1} \in \Lambda_{1}\right\}, H_{1}=U V_{1}$. Because $\theta$ preserves infs and sups, we have that the sets $V\left(\lambda_{1}\right) \quad\left(\lambda_{1} \in \Lambda_{1}\right)$ are pairwise disjoint and non-empty, and $\bar{H}_{1}=Y$.

Next, for each $\lambda_{1} \in \Lambda_{1}$, take a maximal pairwise disjoint collection $v_{2}\left(\lambda_{1}\right)=\left\{V\left(\lambda_{1}, \lambda_{2}\right): \lambda_{2} \in \Lambda_{2}\left(\lambda_{1}\right)\right\}$ of nonempty regular open subsets of $Y$, of $\sigma$-diameters less than $\frac{2}{2}$, and with closures (in $Y$ ) contained in 
$v\left(\lambda_{1}\right)$. Put $v_{2}=U\left\{v_{2}\left(\lambda_{1}\right): \lambda_{1} \in \Lambda_{1}\right\}, H_{2}\left(\lambda_{1}\right)=U v_{2}\left(\lambda_{1}\right)$, $H_{2}=U V_{2}=U\left\{H_{2}\left(\lambda_{1}\right): \lambda_{1} \in \Lambda_{1}\right\}$. By maximality, $H_{2}\left(\lambda_{1}\right)$ is dense in $V\left(\lambda_{1}\right)$, so $H_{2}$ is a dense open subset of $Y$; also $H_{2} \subset H_{1}$. Put $U\left(\lambda_{1}, \lambda_{2}\right)=\theta^{-1}\left(v\left(\lambda_{1}, \lambda_{2}\right)\right) \in R(x), u_{2}\left(\lambda_{1}\right)=\left\{U\left(\lambda_{1}, \lambda_{2}\right): \lambda_{2} \in \Lambda_{2}\left(\lambda_{1}\right)\right\}$, $G_{2}\left(\lambda_{1}\right)=U U_{2}\left(\lambda_{1}\right), G_{2}=U\left\{G_{2}\left(\lambda_{1}\right): \lambda_{1} \in \Lambda_{1}\right\}$. Because $\theta$ is an isomorphism, the sets $U\left(\lambda_{1}, \lambda_{2}\right)$ are non-empty and pairwise disjoint and $G_{2}\left(\lambda_{1}\right)$ is dense in $U\left(\lambda_{1}\right)$, and $G_{2}$ is a dense open subset of $X$.

We next take a maximal pairwise disjoint collection of nonempty regular open subsets $U\left(\lambda_{1}, \lambda_{2}, \lambda_{3}\right) \quad\left(\lambda_{3} \in \Lambda_{3}\left(\lambda_{1}, \lambda_{2}\right)\right)$ of $X$, of $\rho-$ diameters less than $1 / 3$ and with closures contained in $U\left(\lambda_{1}, \lambda_{2}\right)$, and put $\theta\left(U\left(\lambda_{1}, \lambda_{2}, \lambda_{3}\right)\right)=V\left(\lambda_{1}, \lambda_{2}, \lambda_{3}\right)$; and then a maximal pairwise disjoint collection of nonempty regular open subsets $V\left(\lambda_{1}, \lambda_{2}, \lambda_{3}, \lambda_{4}\right)$ of $Y$, of $\sigma$-diameters less than $\frac{1}{4}$ and with closures contained in $V\left(\lambda_{1}, \lambda_{2}, \lambda_{3}\right)$, and put $\theta^{-1}\left(V\left(\lambda_{1}, \lambda_{2}, \lambda_{3}, \lambda_{4}\right)=U\left(\lambda_{1}, \lambda_{2}, \lambda_{3}, \lambda_{4}\right) ;\right.$ and so on, alternately.

For $n=1,2, \ldots$, we write $G_{n}=U\left\{U\left(\lambda_{1}, \ldots, \lambda_{n}\right): \lambda_{n} \in \Lambda_{n}\left(\lambda_{1}, \ldots, \lambda_{n-1}\right)\right.$ $\left.\lambda_{n-1} \in \Lambda_{n-1}\left(\lambda_{1}, \ldots, \lambda_{n-2}\right), \ldots, \lambda_{1} \in \Lambda_{1}\right\}$,

and similarly $H_{n}=$ union of all the sets $V\left(\lambda_{1}, \ldots, \lambda_{n}\right)$. These are dense open subsets of $X, Y$ respectively. Put $A=n\left\{G_{n}: n=1,2, \ldots\right\}$, $B=n\left\{H_{n}: n=1,2, \ldots\right\} ;$ by Baire's Theorem, $A$ and $B$ are dense $G_{\delta}$ subsets of $X, Y$. We define the map $T: A \rightarrow B$ as follows.

Given $x \in A$, we know that $x$ is in $U\left(\lambda_{1}\right)$ for just one $\lambda_{1} \in \Lambda_{1}$, say for $\lambda_{1}=\lambda_{1}(x)$. Similarly $x$ is in just one set $U\left(\lambda_{1}(x), \lambda_{2}(x)\right), \ldots$, just one $U\left(\lambda_{1}(x), \lambda_{2}(x), \ldots, \lambda_{n}(x)\right), \ldots$. Consider the corresponding sets $V\left(\lambda_{1}(x), \lambda_{2}(x), \ldots, \lambda_{n}(x)\right)$ for 
$n=1,2, \ldots$. By construction we have

$$
V\left(\lambda_{1}(x)\right) \supset V\left(\lambda_{1}(x), \lambda_{2}(x)\right) \supset \ldots \supset V\left(\lambda_{1}(x), \lambda_{2}(x), \ldots, \lambda_{n}(x)\right) \supset \ldots .
$$

Further, for each even $n$ we have that the $\sigma$-diameter of $V\left(\lambda_{1}(x), \ldots, \lambda_{n}(x)\right)$ is less than $1 / n$, and that its closure (in $Y$ ) is contained in $V\left(\lambda_{1}(x), \ldots, \lambda_{n-1}(x)\right)$. It follows that

$$
\bigcap_{n=1}^{\infty} V\left(\lambda_{1}(x), \ldots, \lambda_{n}(x)\right)
$$

is a singleton, say $\{y\}$; and we let $T(x)=y$. Clearly $y \in B$, so we have defined a map $T: A \rightarrow B$.

But essentially the same considerations apply with the roles of $X$ and $Y$ interchanged. For each $y \in B$, we have that $y$ is in just one $V\left(\lambda_{1}(y)\right)$, one $V\left(\lambda_{1}(y), \lambda_{2}(y)\right)$, and so on. The corresponding sets $U\left(\lambda_{1}(y), \ldots, \lambda_{n}(y)\right)(n=1,2, \ldots)$ form a decreasing sequence; further for each odd $n$, the $n$th such set has $\rho$-diameter less than $1 / n$ and the closure of the $n$th set is (for $n>1$ ) contained in the $(n-1)$ st . So $\prod_{n=1}^{\infty} U\left(\lambda_{1}(y), \ldots, \lambda_{n}(y)\right)=\{x\}$ for some $x \in A$, and we define $S: B \rightarrow A$ by setting $S(y)=x$. It is clear that the maps $S$ and $T$ are mutually inverse, so $T$ is a bijection of $A$ onto $B$.

To show that $T$ is continuous, suppose $x \in A$ and $\varepsilon>0$ are given; take $n$ even and greater than $1 / \varepsilon$. Then $A \cap U\left(\lambda_{1}(x), \ldots, \lambda_{n}(x)\right)$ is a neighbourhood of $x$ in $A$, and it is mapped by $T$ into a subset of $V\left(\lambda_{1}(x), \ldots, \lambda_{n}(x)\right)$ of $\sigma$-diameter less than $\varepsilon$. Thus $T$ is continuous; and similarly so is $S=T^{-1}$. Hence $T$ is a homeomorphism of $A$ onto $B$.

All that remains is to show that $T$ induces $\theta$, or more precisely, that $T$ induces $j \circ \theta \circ i^{-1}$, where $i$ is the natural isomorphism of $R(X)$ onto $R(A)$ (obtained by intersecting with $A$ ) and $j$ is the analogous isomorphism of $R(Y)$ onto $R(B)$. But it is clear from the construction that $T\left(U\left(\lambda_{1}, \lambda_{2}, \ldots, \lambda_{n}\right) \cap A\right)=V\left(\lambda_{1}, \lambda_{2}, \ldots, \lambda_{n}\right) \cap B$; 
that is, $T$ agrees with $j \circ \theta \circ i^{-1}$ on the sets $U\left(\lambda_{1}, \lambda_{2}, \ldots, \lambda_{n}\right) \cap A$. But these sets form a basis for $R(A)$, in the sense that every (relatively) regular open set $G$ in $A$ is the sup of the sets $U\left(\lambda_{1}, \lambda_{2}, \ldots, \lambda_{n}\right) \cap A$ contained in $i t$, and it follows that $T$ induces $j \circ \theta \circ i^{-1}$, as it should.

COROLLARY 1. Let $\tau$ be an automorphism of $\mathrm{C}(X)$, where $X$ is a complete metric space. Then there exist a dense $G_{\delta}$ subset $C$ of $X$, and a homeomorphism $T^{\prime}$ of $C$ onto itself, such that $T^{\prime}$ induces $\tau$.

For, by the theorem, there are dense $G_{\delta}$ subsets $A, B$ of $X$, and a homeomorphism $T$ of $A$ onto $B$ that induces $\tau$. We put $C_{1}=A \cap B$, $c_{n+1}=T\left(C_{n}\right) \cap T^{-1}\left(C_{n}\right)$ for $n=1,2, \ldots, c=n\left\{C_{n}: n=1,2, \ldots\right\}$, and $T^{\prime}=T \mid C$.

COROLLARY 2. Let $\tau$ be an automorphism of $\mathcal{C}(X)$, where $X$ is a complete metric space. Then there exists a Borel isomorphism (in fact a generalized homeomorphism of class $(2,2)$ ) $T^{\prime \prime}$ of $X$ onto itself, such that both $T$ and $\left(T^{\prime \prime}\right)^{-1}$ take sets of first category to sets of first catego'y, and such that $T^{\prime \prime}$ induces $\tau$.

Apply Corollary 1 , and define $T^{\prime \prime}$ to agree with $T^{\prime}$ on $C$ and with the identity map on $X-C$.

REMARK 1. It is easy to see that the homeomorphism $T$, in the theorem, is essentially unique; in fact, any transformation $T_{1}$ (not necessarily a homeomorphism), that induces (in a reasonable sense) the same isomorphism $\tau$, must agree with $T$ except perhaps on a first category subset of $X$. In particular, $T_{1}$ must itself be a homeomorphism on a residual set. Similar statements apply to the corollaries.

REMARK 2. A similar method applies to the case of a complete isomorphism between $\mathcal{C}(Y)$ and a subalgebra (necessarily complete) of $C(X)$, where $X$ and $Y$ are given complete metric spaces. Say that a subspace $Z$ of $X$ is "thick" in $X$ if it is nowhere of first category; that is, for every non-empty open set $U$ in $X, Z \cap U$ is not of first category in $U$ (or, equivalently, in $X$ ). (Clearly $Z$ residual $\rightarrow Z$ 
thick $\rightarrow Z$ dense in $X$, and Baire's Theorem holds for a thick $Z$.) If we have thick subsets $A$ of $X, B$ of $Y$, where $X$ and $Y$ are given complete metric spaces, and a continuous (or more generally Borel measurable) map $T: A \rightarrow B$ such that $T^{-1}$ takes each first category subset of $B$ to a first category subset of $A$, then $T^{-1}$ induces, in a natural way, a complete isomorphism $\tau: \mathcal{C}(Y) \rightarrow \mathcal{C}(X)$. Conversely it can be shown that, given a complete isomorphism $\tau: \mathcal{C}(Y) \rightarrow \mathcal{C}(X)$, there exist thick subsets $A$ of $X, B$ of $Y$ (in fact $A$ can be made residual in $X$ ) and a continuous surjection $T: A \rightarrow B$ such that $T^{-1}$ preserves first category, and such that $T^{-1}$ induces $\tau$.

\section{References}

[1] J.R. Choksi, "Automorphisms of Baire measures on generalized cubes. II", Z. Wahrscheinlichkeitstheorie und verw. Gebiete 23 (1972), 97-102.

[2] John C. Oxtoby, Measure and category. A survey of the analogies between topological and measure spaces (Graduate Texts in Mathematics, 2. Springer-Verlag, New York, Heidelberg, Berlin, 1971).

[3] D. Maharam, "Category, boolean algebras and measure", General topology and its relations to modern analysis and algebra IV. Part A: Invited papers, 124-135 (Proc. Fourth Prague Topological Sympos. 1976. Lecture Notes in Mathematics, 609. Springer-Verlag, Berlin, Heidelberg, New York, 1977).

[4] J.v. Neumann, "Einige Sätze über messbare Abbildungen", Ann. of Math. (2) $33(1932), 574-586$.

Department of Mathematics, Institute of Advanced Studies, Australian National University, Canberra, ACT;

Department of Mathematics, University of Rochester, Rochester, New York, USA. 